\title{
RESEARCH PAPER \\ PREVALENCE OF MYOPIA AMONGST PATIENTS WITH REFRACTIVE ERROR IN THE KUMASI METROPOLIS OF GHANA
}

\author{
${ }^{\mathbf{1}}$ N. Y. Koomson, ${ }^{2}$ S. Y. Lartey, and ${ }^{\mathbf{3}}$ K. K. Adjah, \\ ${ }^{1}$ Department of Optometry and Visual Science, KNUST, Kumasi. \\ ${ }^{2}$ Departemnt of Eye, Ear, Nose and Throat, School of Medical Sciences, KNUST, Kumasi \\ ${ }^{3}$ Department of Optometry, University of Kwazulu-Natal, \\ South Africa
}

\begin{abstract}
This paper sought to report on the prevalence of myopia and its distribution pattern among individuals who seek eye health in eye care facilities in Kumasi. In a retrospective cross sectional study, the two leading major public eye facilities (Komfo Anokye Teaching Hospital and Kumasi South hospital) were purposively selected whilst simple random sampling method was used to select five of the private eye care facilities. In total, 6027 records of subjects with refractive errors who visited the eye facilities between January 2010 and December 2010 were reviewed. The results revealed that $54.12 \%$ of the patients records reviewed were myopic. Of the 3263 myopes, $75.45 \%$ were low myopes, $15.78 \%$ were moderate myopes and $8.77 \%$ were high myopes. The prevalence of myopia among the male study population was $58.68 \%$ and $50.31 \%$ among the female subjects. The study concludes that myopia is the most common refractive error that is presented for refraction in the eye facilities in Kumasi. More community based studies are required to determine the actual burden of myopia and to explore the gender bias in the larger population.
\end{abstract}

Keywords: Myopia, Ametropia, Prevalence

\section{INTRODUCTION}

Myopia or short sightedness is a condition in which the eye does not see sharply because distant objects are not brought to a focus on the retina but rather in front of it. It is the most prevalent eye defect in the world affecting about $41.6 \%$ of the United States of America's adult population (Vitale et al, 2009), and 50\% to $80 \%$ of South Eastern Asians (Lin et al., 1988; Hosaka, 1988). Its prevalence is reported to be increasing in both developed and developing countries (Mutti and Zadnik, 1995; Hammond et al., 2001).

Rays of light normally travel from distant objects ( 6 metres and beyond) in parallel lines, and when they come into contact with an emmetropic (normal) eye, they come to a sharp focus on the retina (film of the eye) forming a sharp image of the distant object, without the 


\section{Koomson et al.}

need for the eye to activate its accommodative (focusing) system to fine tune the image. However, in most eyes, the image fails to come to a sharp focus on the retina when the accommodative system is relaxed. This state of the eye is known as ametropia or refractive error. It is further sub classified based upon where the image comes to sharp focus. Myopia is a refractive status of the eye in which parallel rays of light from a distant object come to focus in front of the retina with accommodation relaxed. This therefore results in the blurriness of distant objects which improves as the object is brought closer to the eyes hence, the common name short sightedness. Myopia has been classified in various ways such as by cause, clinical entity, degree and age of onset. Numerous researches have also been undertaken to determine the causes, risk factors, distribution, prevalence and incidence of myopia. Results from these studies vary widely across regions and even within same due to differences in the genetic predisposition, environmental conditions and ways of life across regions. Another reason was lack of uniformity for the definition of myopia across studies.

Racial variations in myopia prevalence are reported among Australian Aboriginals and Australians of European descent as indigenous population have lower refractive error cases (Taylor, 1981). In The United States of America, the Baltimore eye survey reported a myopia prevalence (spherical equivalence of $-0.50 \mathrm{D}$ ) of $19.4 \%$ among blacks and $28.1 \%$ among whites (Katz et al, 1997).

In Ghana, the prevalence of myopia among school children has been reported as $1.7 \%$ in Agona Swedru (Ovenseri-Ogbomo and Assien, 2010) and $7 \%$ in Accra (Ntim-Amponsah, 2007). Hospital based studies in the central region of Ghana and Ille-Ife in the Osun State of Nigeria reported prevalence estimates of 29.2\% (Ovenseri-Ogbomo, and Morny, 2008) and $22.7 \%$ respectively (Adegbehingbe et al., 2003).
To the best of our knowledge, there is no documented study that shows the prevalence and distribution patterns of the different degrees of myopia in Kumasi, Ghana. The purpose of this study was to report on the prevalence of myopia and its patterns of distribution among individuals who seek eye health in eye care facilities in Kumasi.

\section{METHODS}

This was a retrospective cross sectional study of the records of patients. The study was conducted in 2011 in the Kumasi Metropolis, the second largest city in Ghana which is located in the Central part of the country. It has a population of $1,468,609$. The city has a number of eye care facilities in both the public and private sectors. Among these are the two leading public eye facilities, Komfo Anokye Teaching Hospital (KATH) and Kumasi South Hospital and 15 private eye care facilities. The public hospitals are managed by ophthalmologists, whilst the private ones are managed by either optometrists or ophthalmologists.

The two leading major public eye facilities were purposively selected whilst simple random sampling method was used to select five out of the 15 private eye care facilities.

Ethical clearance was obtained from the ethical committee of the School of Medical Sciences at the Kwame Nkrumah University Science and Technology.

Records of all 2,803 patients who received eye care from the five randomly selected private eye care facilities between January 2010 and December 2010 were selected. The records of 3,147 and 1,050 patients were randomly selected from those who visited the eye care units at the Komfo Anokye Teaching Hospital and the Kumasi South hospital respectively between January 2010 and December 2010.

Patients documented to be suffering from glaucoma, corneal and external eye diseases, diabetics, cataract, maculopathy, post-operative re- 
fractive errors, tropia, anisometropia of 1D or more and records with incomplete information were excluded. A total of $6,027(86.1 \%)$ patient records met the inclusion criteria and were included in this retrospective study.

Information extracted from each case record included sex, age, distance visual acuity (with and without spectacle correction), funduscopic findings, autorefraction and/or subjective refraction results. $90 \%$ of the children aged 5 years to 15 years and $20 \%$ of the subjects aged 16 years to 25 years had both cycloplegic and subjective refraction results recorded. The cycloplegic agents used by examiners were different. The two public hospitals had used atropine $1 \%$ whiles the five private facilities used a combination of cyclopentolate $1 \%$ and a drop of $0.5 \%$ tropicamide.

For the purpose of this study, myopia was classified into: low myopia i.e.- 0.50 to $-2.25 \mathrm{DS}$, moderate myopia -2.50 to $-4.75 \mathrm{D}$ and high myopia $-5.00 \mathrm{D}$ and less. Any other refractive status including astigmatism beyond -1.00 was classified as "other". The analysis of data was performed with Microsoft Office Excel 2007 and SPSS 16.

\section{RESULTS}

\section{Demographic Data}

In total, 6,027 records of subjects with refractive errors only were reviewed, and the age distribution of the subjects whose records were reviewed is shown in table 1 . A high correlation of 0.94 between the spherical equivalence refractive error of the right and left eyes was found and so the refractive status of the right eye only was used. The age (in years) distribution of the subjects ranged from 5 to 84 , with a mean age of 36.68 years and standard deviation of 18.73 .

Of the 6027 subjects with refractive errors, $54.22 \%$ were females whilst the remaining $45.78 \%$ were males (table 2). The mean age of females was 36.08 years with standard deviation of 18.89 , and among the male subjects were 37.39 years and 18.53 respectively $\mathrm{P}=0.0068$.

\section{Prevalence of Myopia}

Of the 6,027 subjects' records reviewed, 3,263 $(54.12 \%)$ were diagnosed as having myopia ($0.50 \mathrm{D}$ to $-20.00 \mathrm{D})$. The remaining 2,764 (45.88\%), who were diagnosed as either emmetropic, hyperopic or astigmatism beyond 1.00 , were classified as "Other".

Of the 3263 myopes, $75.45 \%$ were low myopes, $15.78 \%$ were moderate myopes and $8.77 \%$ were high myopes (refer to fig 1 ). The prevalence of low, moderate and high myopia in the sampled population are $40.85 \%, 8.54 \%$ and $4.75 \%$ respectively.

Overall, the prevalence of myopia among the male study population was $58.68 \%$ (95\% CI $56.82-60.53 \%)$ and $50.31 \%$ (95\% CI 48.58 $52.03 \%$ ) among the female subjects $\mathrm{P}=\mathbf{0 . 0 0}$. (see fig 2).

Table 3 shows the prevalence of myopia among the various age groups with the highest prevalence among 20-29 years age range and lowest among those aged 70 years and above.

Table 1: Age distribution of patients

\begin{tabular}{ccc}
\hline Age & Frequency & Percentage \\
\hline $0-9$ & 160 & 2.7 \\
$10-19$ & 1122 & 18.6 \\
$20-29$ & 1537 & 25.5 \\
$30-39$ & 530 & 8.8 \\
$40-49$ & 761 & 12.6 \\
$50-59$ & 1115 & 18.5 \\
$60-69$ & 493 & 8.2 \\
70 and above & 309 & 5.1 \\
Total & $\mathbf{6 0 2 7}$ & $\mathbf{1 0 0 . 0}$ \\
\hline
\end{tabular}


Table 2: Age distribution by gender of patients

\begin{tabular}{cccc}
\hline \multirow{2}{*}{ Age group } & \multicolumn{2}{c}{ Gender $(\%)$} & Total (n) \\
& Female & Male & \\
\hline $0-9$ & 62.50 & 37.50 & 160 \\
$10-19$ & 62.03 & 37.97 & 1122 \\
$20-29$ & 50.49 & 49.51 & 1537 \\
$30-39$ & 46.79 & 53.21 & 530 \\
$40-49$ & 47.83 & 52.17 & 761 \\
$50-59$ & 61.35 & 38.65 & 1115 \\
$60-69$ & 51.93 & 48.07 & 493 \\
70 and above & 46.60 & 53.40 & 309 \\
Total & $\mathbf{5 4 . 2 2}$ & $\mathbf{4 5 . 7 8}$ & $\mathbf{6 0 2 7}$ \\
\hline
\end{tabular}

Table 3: Age distribution of myopia

\begin{tabular}{lc}
\hline Age Group & Prevalence of Myopia (\%) \\
\hline $0-9$ & 47.50 \\
$10-19$ & 69.25 \\
$20-29$ & 75.80 \\
$30-39$ & 64.53 \\
$40-49$ & 39.29 \\
$50-59$ & 28.88 \\
$60-69$ & 31.03 \\
70 and above & 41.75 \\
\hline
\end{tabular}

\section{DISCUSSION}

The overall prevalence of myopia in this study was $54.12 \%$, a figure which is higher than most population and hospital based studies. Information gathered from studies of selected groups in hospitals and schools may not be representative of the total population as it is limited by selection bias due to self-referral and inconsistent data from unscientific recording procedures. For instance the study by Midelfat et al., (1992) reported a prevalence estimate of $50.3 \%$ among 133 medical students in Norway, a figure which is close to the prevalence estimate found in this study even though they reported on a smaller sample size. A hospital based study in Yenagoa, Bayelsa State in Nigeria, reported a lower prevalence of $28.9 \%$ (Azonobi, 2010). However, in Azonobi's study myopes with astigmatism beyond -0.25 were classified as astigmates, hence the lower prevalence. A tertiary hospital in Pakistan found $60 \%$ prevalence of myopia among phakic patients (Ashok et al, 2012), a figure which is slightly higher than what this study found. This is however not surprising as Pakistanis are Asians and have been shown to be ethnically susceptible to myopia.

The prevalence of low, moderate and high myopia in the study population was $40.85 \%$, $8.54 \%$ and $4.75 \%$ respectively. A study conducted by Vitale et al., (2009) in the USA also showed a trend similar to that found in this study.

Reports from studies comparing the prevalence of myopia between males and females have been equivocal; it appears to vary from population to population. Whiles some studies have reported no difference between sexes, others have reported otherwise. In this study the prevalence estimate of males is higher than females which are in contrast with a number of studies that report of a higher prevalence in girls than in boys. (Zadnik et al., 2003; Ip et al., 2008; Rudnicka et al., 2008). The reason for sex-related difference is not certain but could be due to the relative differences in response to health seeking behaviour. It could also be that males do more jobs requiring corrected myopia than females.

The prevalence estimates among the 10-19 and 20-29 age groups are relatively higher because school myopia develops at age 10 years and progresses into early adulthood (Ware, 1813; 
Prevalence of myopia in the Kumasi Metropolis ... 77



Fig. 1: Distribution of myopia of severity

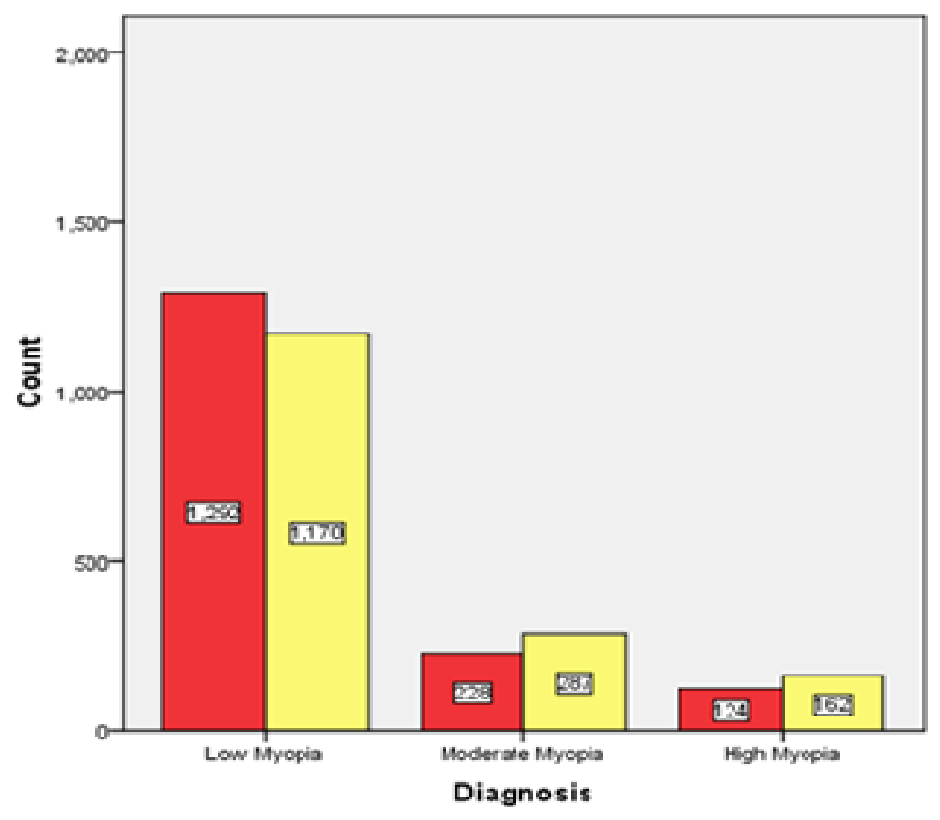

Fig. 2: Gender distribution of myopia 
Koomson et al.

Angle and Wissman, 1980; Zadnik and Mutti, 1987). These adolescents however require good distant vision to engage in social activities. We should therefore expect that those in this age group would be more frequent at refractive clinics. Between 25 and 40 years, myopia is stable so we would not expect those in that age group to be reporting unless they want to change their frames.

Among the 20-29 year olds the prevalence of myopia is at its peak because myopia progression continues in early adulthood from 19 years to 29 years when the lifestyle of individuals need extensive near work as shown in law and engineering students and microscopist (Zadnik and Mutti, 1987; McBrien and Adams, 1997; Kinje et al., 2000; Bullimore et al, 2002). In a retrospective study conducted among 8 to 85years in Nigeria found the prevalence of myopia to peak from 16 to 25years (Ayanniyi et al., 2010). The distribution patterns of refractive error could account for the difference between the two retrospective studies.

During the analysis of data collected, it was observed that $20 \%$ of subjects aged between 16 years and 25 years had undergone cycloplegic refraction. This was significant as cycloplegic refraction is mostly carried out on children and rarely in young adults. It was revealed from further correspondence with clinicians from study sites, that cycloplegic refraction is a standard procedure carried out among all young adults who complain of headaches and eyestrains with the use of the eyes. This helps the clinicians to diagnose pseudomyopia and other accommodative and binocular vision anomalies.

\section{LIMITATIONS OF STUDY}

This study is a cross sectional retrospective study of the clinical records of patients who visited eye care facilities in Kumasi, Ghana. The patients record charts contained data not recorded for research purposes and so might lack a number of relevant data on pathology that might be relevant for exclusion or inclu- sion. Secondly, the prevalence estimate of myopia in this study might be overestimated as the study was done on a motivated population who had self-referred. Thus the findings from this study cannot be generalised for the entire population.

\section{CONCLUSION}

Myopia is the most common refractive error that is presented for refraction in the eye facilities in Kumasi. Low myopia affects more people who report for examination than moderate and high myopia. Myopia was found to be more prevalent in males than in females. Community based population studies are required to determine the actual burden of myopia and to explore this gender bias in the larger population.

\section{REFERENCES}

Adegbehingbe, B. O., Majekodunni, A. A., Akinsola, F. B., and Soetan, E. O. (2003). Pattern of refractive errors at Obafemi Awolowo University Teaching Hospital, IlleIfe, Nigeria. Nigerian Journal of Ophthalmology. 11: 76-79

Angle, J. and Wissman, D. A. (1980). The epidemiology of myopia. American Journal of Epidemiology; 111: 220-228.

Ashok, K. N., Shafi, M. J., Mohan, P. and Khairuddin, M. (2012). Incidence of refractive error and amblyopia among young adults - A hospital based study. In: S. Rumelt, ed. 2012. Advances in Ophthalmology. InTech. Ch.8.

Ayanniyi, A. A., Adepoju, F. G., Ayanniyi, R. O. and Morgan, R. E. (2010). Challenges, attitudes and practices of the spectacle wearers in a resource-limited economy. BMC Ophthalmology. 10: 83-87

Azonobi, I. R. (2010). Refractive error blindness in Yenagoa, Bayelsa State, Nigeria: A hospital based study. Benin Journal of Postgraduate Medicine. 12: 37-42. 
Bullimore, M. A., Jones, L. A., Moeschberger, M. L., Zadnik, K., and Payor, R. E. (2002). A retrosceptive study of myopia progression in adult contact lens wearers. Investigative Ophthalmology and Visual Optics. 43: 21102113

Hammond, C. J., Snieder, H., Gilbert, C. E., and Spector, T. D. (2001). Genes and environment in refractive error: the twin eye study. Investigative Ophthalmology and Visual Science. 42: 1232-1236

Hosaka, A. (1988). Population studies: myopia experience in Japan. Acta Ophthalmology Suppl. 185: 37-40

Ip, J. M., Huynh, S. C., Robaei, D., Kifley, A., Rose, K. A., Morgan, I. G., Wang, J. J. and Mitchell, P. (2008). Ethnic difference in refraction and ocular biometry in a populationbased sample of 11-15 year old Australian children. Eye. 22: 649-656.

Katz, J., Tielsch, J. M., and Sommer, A. (1997). Prevalence and risk factors for refractive errors in an adult inner city population. Investigative Ophthalmology and Visual Science. 38: 334-340

Kinje, B., Midelfat, A., Jacobsen, G., and Rystad, J. (2000). The influence of near-work on development of myopia among university students: a three-year longitudinal study among engineering students in Norway. Acta Ophthalmologica. $78: 26-29$.

Lin, L. L., Chen, C. J., Hung, P. T., and Ko, L. S. (1988). Nation-wide survey of myopia among school children in Taiwan, 1986. Acta Ophthalmol Suppl. 185: 29-33

McBrien, N. A., and Adams, D. W. (1997). Longitudinal investigation of adult -onset and adult progression of myopia in an occupational group. Refractive and biometric findings. Investigative Ophthalmology and Visual Science. 38: 321-33
Midelfat, A., Aamo, B., Sjohang, K. A. and Dysthe, B. E., (1992). Myopia among students in Norway. Acta Ophthalmologica. 70: 317-322

Mutti, D. O., and Zadnik, K. (1995). The utility of three predictors of childhood myopia: A Bayesian analysis. Vision Research, 35(9), $1345-1352$

Ntim-Amponsah, C. T. (2007). Contribution of refractive errors to visual impairment in patients at Korle-Bu teaching Hospital. Ghana Medical Journal; 41(2) , 68-71

Ovenseri-Ogbomo G. O., and Assien, R. (2010). Refractive error in school children in Agona Swedru, Ghana. 69 (2), 86-92

Ovenseri-Ogbomo, G. and Morny, E. K. (2008). Cause of visual impairment in Central Region, Ghana. Journal of Nigerian Optometric Association; 14:11-13

Rudnicka, A. R., Owen, C. C., Richards, M., Wadsworth, M. E., and Strachan, D. P. (2008). Effect of breastfeeding and sociodemographic factors on visual outcome in childhood and adolescence. American Journal of Clinical Nutrition. 87: 1392-1399

Taylor, H. R. (1981). Racial variations in vision. American Journal of Epidemiology. 113: $62-80$.

Vitale, S., Sperduto, R. R., Ferris, F. L. (2009). Increased prevalence of myopia in the United States between 1971-1972 and 1999-2004; Archives of Ophthalmology. 127: 1632-1639

Ware, J. (1813). Observations relative to the near and distant sight of different persons. Philosophical Transaction of the Royal Society of London. 103: 31-50

Zadnik, K., Manny, R. E., Yu, J. A. Mitchell, G., Cotter, S. A., Quiralte, J. C.; Shipp, M. D., Friedman, N. E., Kleinstein, R. N., 


\section{Koomson et al.}

Walker, T. W., Jones, L. A., Moeschberger, M. L. and Mutti, D. O. (2003). Ocular com-

Zadnik, K., and Mutti, D. O (1987). Refractive ponent data in school children as a function of age and gender. Optometry and Vision Science. 80:226-236

error changes in law students, American Journal of Optometry and Physiological Optics. 64: 558-561 